\title{
Nutzen und Grenzen der nationalen Patientenzufriedenheitsbefragung
}

\author{
Das Schweizer Gesundheitssystem ist zugleich eins der teuersten und leistungs- \\ stärksten der Welt. Die Bevölkerung ist mit dem Gesundheitssystem sehr zufrieden. \\ Um die Qualitätsentwicklung weiter zu fördern wird die Patientenzufriedenheit im \\ stationären Setting systematisch erhoben und publiziert. Dieses Instrument hat \\ neben seinen Möglichkeiten aber auch Grenzen.
}

\section{Stephanie Hellweg}

Fachexpertin Physiotherapie Neurologische Rehabilitation, MSc Neurorehabilitation, Dipl. Physiotherapeutin

Interessenverbindungen Die Autorin ist in der Rehaklinik Bellikon tätig, die an der nationalen Erhebung der Patientenzufriedenheit teilnimmt.

\footnotetext{
* Die Literatur findet sich online unter www.saez.ch $\rightarrow$ Aktuelle Ausgabe oder Archiv $\rightarrow 2014 \rightarrow 50$
}

Korrespondenz: Stephanie Hellweg Rehaklinik Bellikon Mutschellenstrasse 2 CH-5454 Bellikon Tel. 0564855683 Fax 0564855257 stephanie.hellweg[at] rehabellikon.ch
In der Schweiz werden seit einigen Jahren verschiedene Qualitätsparameter systematisch auf nationaler Ebene erfasst und publiziert, um die Leistungserbringer in ihren Bemühungen um kontinuierliche Verbesserung zu unterstützen. Für den Bereich der stationären Krankenversorgung koordiniert der Nationale Verein für Qualitätsentwicklung in Spitälern und Kliniken (ANQ) das Vorgehen in den Bereichen Akutsomatik, Rehabilitation und Psychiatrie. Neben objektiven Parametern, zum Beispiel Raten von unerwünschten Ereignissen wie Wundinfekten, Reoperationen und Stürzen in der Akutsomatik, sowie Verbesserungen der Funktionsfähigkeit in der Rehabilitation, besitzt die Patientenzufriedenheit, welche die subjektive Sichtweise der Patienten wiedergibt, einen hohen Stellenwert. Diese Gewichtung entspricht internationalen Gepflogenheiten, wo die Patientenzufriedenheit als anerkannter Qualitätsindikator akzeptiert ist und für Outcomeforschung sowie Benchmarking verwendet wird [1]*.

\section{Messung der Patientenzufriedenheit auf nationaler Ebene}

Patientenzufriedenheit wird häufig als Beurteilung aller Aspekte der medizinischen Versorgung aus Sicht der Patienten verstanden und umfasst somit sowohl die zwischenmenschliche Beziehungsgestaltung als auch die organisatorischen Abläufe [2]. Diese Merkmale machen die Patientenzufriedenheit vielschichtig und multidimensional und entziehen sie somit einer allgemeingültigen operationalisierenden Definition [3].

ANQ erhebt die Patientenzufriedenheit anhand von 5 Fragen, die im Expertenkonsens gefunden wurden. Die Fragen lauten:

\section{Fragen Akutsomatik}

1. Würden Sie für dieselbe Behandlung wieder in dieses Spital kommen?

2. Wie beurteilen Sie die Qualität der Behandlung, die Sie erhalten haben?

\section{Utilité et limites de l'enquête natio-} nale sur la satisfaction des patients

En novembre 2014, l'Association nationale pour le développement de la qualité dans les hôpitaux et les cliniques (ANQ) a publié, pour la première fois dans le domaine de la réadaptation, les résultats de l'enquête nationale sur la satisfaction des patients. Des chiffres collectés de manière analogue ont déjà été publiés en 2013 pour le domaine des soins somatiques aigus. Dans l'ensemble, les chiffres montrent que les patients des hôpitaux et cliniques suisses sont très satisfaits. Bien que la satisfaction des patients est un indicateur de qualité répandu aussi bien pour la recherche sur les résultats que pour comparer les hôpitaux et les cliniques, il s'agit de se demander ce que l'on évalue vraiment, ce que le patient peut réellement évaluer et quels sont les facteurs susceptibles d'influencer les résultats. S'il est évident que le feed-back des patients peut avoir une influence positive sur la qualité du service dans les hôpitaux et les cliniques, le recours à la gestion de la qualité en médecine représente une réelle plus-value sur le plan des relations humaines. En effet, la perception du rôle des soignants et des patients a fortement évolué au cours des dernières années. Les patients sont de plus en plus souvent associés aux décisions, et ont donc des attentes plus élevées à l'égard des prestations dont ils bénéficient. Cependant, il convient de remarquer que les patients peuvent difficilement évaluer la qualité technique de la prestation et que la satisfaction des patients ne constitue donc pas un indicateur de la qualité médicale. 
Abbildung 1

Likert-Skala für die Antwort der Patienten.

\section{Würden Sie für dieselbe Behandlung wieder in dieses Spital kommen? Bitte die für Sie zutreffende Zahl ankreuzen.}

(1) (1) (2) (4) (5) (6) (7) (9) (10)
auf keinen Fall

3. Wenn Sie Fragen an einen Arzt oder eine Ärztin stellten, bekamen Sie verständliche Antworten?

4. Wenn Sie Fragen an das Pflegepersonal stellten, bekamen Sie verständliche Antworten?

5. Wurden Sie während Ihres Spitalaufenthaltes mit Respekt und Würde behandelt?

\section{Fragen Rehabilitation}

1. Würden Sie für dieselbe Behandlung wieder in diese Rehabilitationsklinik kommen?

2. Wie beurteilen Sie die Qualität der Rehabilitationsbehandlung, die Sie erhalten haben?

3. Wenn Sie Fragen an Ihre Ärztin oder Ihren Arzt stellten, bekamen Sie verständliche Antworten?

4. Wie fanden Sie die Betreuung durch das therapeutische Personal, durch das Pflegepersonal und den Sozialdienst während Ihres Aufenthaltes?

5. Wurden Sie während Ihres Rehabilitationsaufenthaltes mit Respekt und Würde behandelt?

(Quelle: [4])

Die Fragen haben nicht den Anspruch, alle Ebenen der Patientenzufriedenheit abzudecken, sondern fokussieren bewusst auf wenige, laut ANQ für alle Spitäler und Kliniken relevante Themen. Sie betref-

\section{Abbildung 2}

Funnel-Plot für die Frage nach der Betreuung.

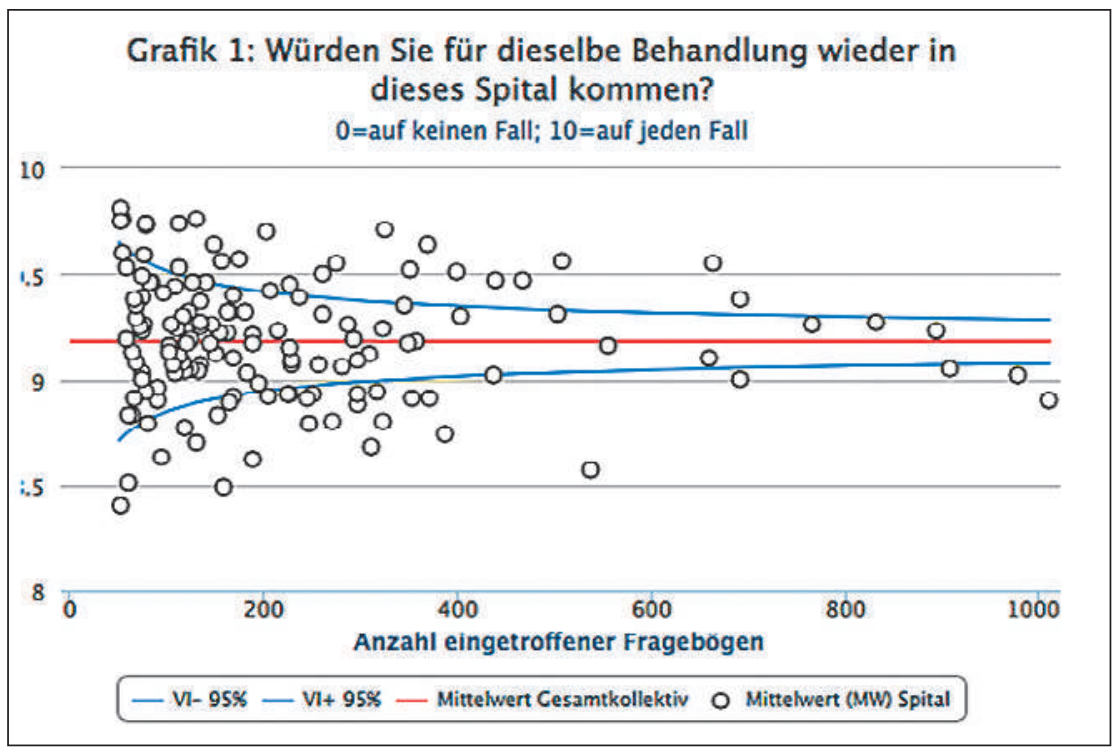

fen die Rückkehrwilligkeit, die Qualität der Behandlung, die Verständlichkeit der ärztlichen Auskünfte, die Beachtung von Respekt und Würde und, ausschliesslich in der Akutsomatik, die Verständlichkeit der pflegerischen Auskünfte, respektive ausschliesslich in der Rehabilitation, die Qualität von Pflege, Therapien und Sozialberatung [4, 5]. Mit dieser Auswahl entsprechen die Fragen den wichtigsten Dimensionen, die in einer Metaanalyse als relevant identifiziert wurden [6].

Die Patienten geben ihre Antworten auf einer 11-stufigen sogenannten Likert-Skala mit den Werten 0 bis 10 .

Die Veröffentlichung erfolgt für jede Frage als klinikspezifischer Durchschnittswert in einem Funnelplot (Abb. 2), der das Durchschnittsergebnis und das 95\%-Konfidenzintervall darstellt.

In dieser Weise veröffentlicht wurden bis heute die Befragungen 2012 in der Akutsomatik und 2013 in der Rehabilitation.

\section{Die Ergebnisse}

Sowohl in der Akutsomatik [7] als auch in der Rehabilitation [5] schwankten die Durchschnittsergebnisse gemittelt über alle Kliniken in allen Fragen um den Wert 9. Hieraus kann man schlussfolgern, dass Patienten grundsätzlich sehr zufrieden mit ihrem Aufenthalt im Spital oder der Klinik sind. Natürlich liegen die Durchschnittsergebnisse einzelner Spitäler und Kliniken für einzelne Fragen ausserhalb des Konfidenzintervalls. Bei Abweichungen nach unten werden die Verantwortlichen sich fragen, was die Ursache ist. Die Erklärungen für den Effekt können sowohl spital-/klinikspezifisch als auch methodischer Natur sein.

\section{Der Nutzen}

Der unmittelbare Nutzen der Zufriedenheitsbefragung liegt in der Suche nach klinikspezifischen Verbesserungsmassnahmen und deren Umsetzung mit dem Ziel, die Zufriedenheit der Patienten zu erhöhen. Die Vergleichbarkeit der Ergebnisse auf nationaler Ebene gekoppelt mit der hohen Transparenz, die durch die Veröffentlichung im Internet entsteht, mag den Wettbewerb unter Spitälern und Kliniken erhöhen. Dieses kann Handlungsdruck entstehen lassen, was im Sinne der Patienten grundsätzlich zu begrüssen ist. Eine positive Auswirkung auf die zwischenmenschliche Beziehungsgestaltung und die Servicequalität ist zu erwarten. Diese wird wiederum, als positive Rückkopplung für Spitäler und Kliniken, die Patientenbindung erhöhen.

Weiterhin zeigen Studien, dass die wahrgenommene Zufriedenheit die Qualität der medizinischen Betreuung beeinflussen kann [8]. Insbesondere können Resultate aus Befragungen dazu beitragen, Problembereiche zu identifizieren und Massnahmen aufzugleisen [3], so dass die Möglichkeit besteht, dass Spitäler und Kliniken durch Verbesserung ihrer 
Patientenzufriedenheit auch die medizinische Versorgungsqualität positiv beeinflussen.

Generell ist abzusehen, dass qualitätssichernde Massnahmen in Spitälern und Kliniken einen Bedeutungszuwachs erfahren. Durch diese qualitätssichernden Massnahmen hat sich in den letzten Jahren das Rollenverständnis der Behandelnden gegenüber den Patienten gravierend geändert. Patienten werden als mitentscheidende Partner gewonnen, die bestimmte Erwartungen an die Dienstleistungen haben. Analog zu anderen Bereichen der personenbezogenen Dienstleistungen kann man in der Rehabilitation vom Co-Produktionsprinzip sprechen, was unterstreicht, dass die Leistung in der direkten Interaktion mit den Patienten entsteht, d.h., die Leistung der Erbringer kann nicht getrennt von der Leistung der Patienten betrachtet werden. Während in der

\section{Auch der momentane Gesundheitszustand und} der Sozialstatus beeinflussen die Zufriedenheit.

Medizin früher Begriffe wie Compliance verwendet wurden, was besagt, dass der Patient Verhaltensregeln oder Vorschläge des Arztes befolgt und somit auch hauptverantwortlich für deren Umsetzung ist, werden heute eher Begriffe wie Adhärenz oder Konkordanz gewählt, die betonen, dass es um gegenseitig vereinbarte Massnahmen oder einen Behandlungsplan geht. Der Nutzen der Patientenzufriedenheitsbefragung liegt also auch darin, dass Patienten noch stärker als Partner wahrgenommen werden. rend nur ein kleiner Teil der Varianz durch z. B. die Spital- oder Abteilungsebene erklärbar ist [11].

Ein Gütekriterium, das insbesondere in der öffentlichen Berichterstattung eine enorme Bedeutung hat, ist die Risikoadjustierung. Eine Risikoadjustierung hat den Sinn, dass die Ergebnisse tatsächlich die Behandlungsqualität und nicht den sogenannten "Case-Mix» des bewerteten Leistungserbringers wiedergeben. Zum Beispiel ist bekannt, dass Universitätsspitäler im Durchschnitt Patienten betreuen, deren Gesundheitszustand schlechter ist und die mehr Komorbiditäten aufweisen als in kleineren Spitälern. Diesen Patientenmerkmalen wie Krankheitsschwere, Alter, Geschlecht, aber auch Patientenpräferenzen, die nicht gleichmässig auf alle Leistungserbringer verteilt sind, muss Rechnung getragen werden. In den Niederlanden sind folgende CaseMix-Variablen ermittelt worden, für die eine Risikoadjustierung empfohlen wird: Alter, Gesundheitszustand und Bildungsstand [11]. Für die Schweiz sind diese Empfehlungen derzeit noch ausstehend.

Der ANQ gibt an, seine Daten für Alter, Geschlecht und Versicherungsstatus als primäre Variablen zu korrigieren [7]. Zusätzlich wurden in der Analyse Akutsomatik 2012 Daten verwendet, welche die Spitäler routinemässig an das Bundesamt für Statistik gemeldet hatten [7]. Somit werden zwar viele Variablen ermittelt, jedoch fehlen relevante Variablen, wie z. B. Diagnosen bzw. der Schweregrad der Erkrankung, die einen Einfluss auf das Ergebnis haben könnten.

Eine Vielzahl methodischer Aspekte hat einen Einfluss auf das Ergebnis von Zufriedenheitsmessungen. Zum Beispiel beeinflusst der Zeitpunkt der Erhebung die Zufriedenheit: Je kürzer der Zeitraum zwi-

\section{Der Nutzen der Patientenzufriedenheitsbefragung liegt auch darin, dass Patienten noch stärker als Partner wahrgenommen werden.}

\section{Systematische Einflüsse auf die Patienten- zufriedenheit und Risikoadjustierung}

Viele verschiedene Faktoren haben nachweislich einen Einfluss auf die Patientenzufriedenheit. So sind zum Beispiel ältere Patienten in der Regel zufriedener als jüngere und Männer sind in einigen Aspekten zufriedener als Frauen [9]. Ein höherer Bildungsabschluss, ein langer Spitalaufenthalt und vorherige Aufenthalte im selben Spital senken die generelle Zufriedenheit [9]. Auch der momentane Gesundheitszustand [10] und der Sozialstatus beeinflussen die Zufriedenheit. Diese Einflüsse sind so stark, dass einige Experten die Meinung vertreten, dass ein substantieller Anteil der Varianz der Patientenzufriedenheit auf die Patientenebene zurückzuführen ist, wäh- schen der Zufriedenheitsmessung und dem Austritt war, desto zufriedener sind Patienten [12, 9]. Allgemein lässt sich beobachten, dass zufriedene Patienten eher auf Zufriedenheitsbefragungen antworten als unzufriedene Patienten, was zu einer systematischen Verzerrung oder Bias führen kann [3]. Sowohl die Art der Fragen, ob eher auf einer Messskala beurteilt wird oder ob Ja/Nein-Fragen gestellt werden [13], als auch die Anordnung der Fragen können die Zufriedenheit beeinflussen. Letzteres kann auch in der nationalen Zufriedenheitsbefragung systematisch genutzt werden. Durch die Auswahl und Anordnung von Zusatzfragen, was den Spitälern und Kliniken erlaubt ist, kann das Ergebnis systematisch verzerrt werden. In der Rehabilitation wurden die 
Abbildung 3

Ob ein Patient zufrieden mit dem Spitalaufenthalt ist, hängt nicht nur von der medizinischen Betreuung $a b$.

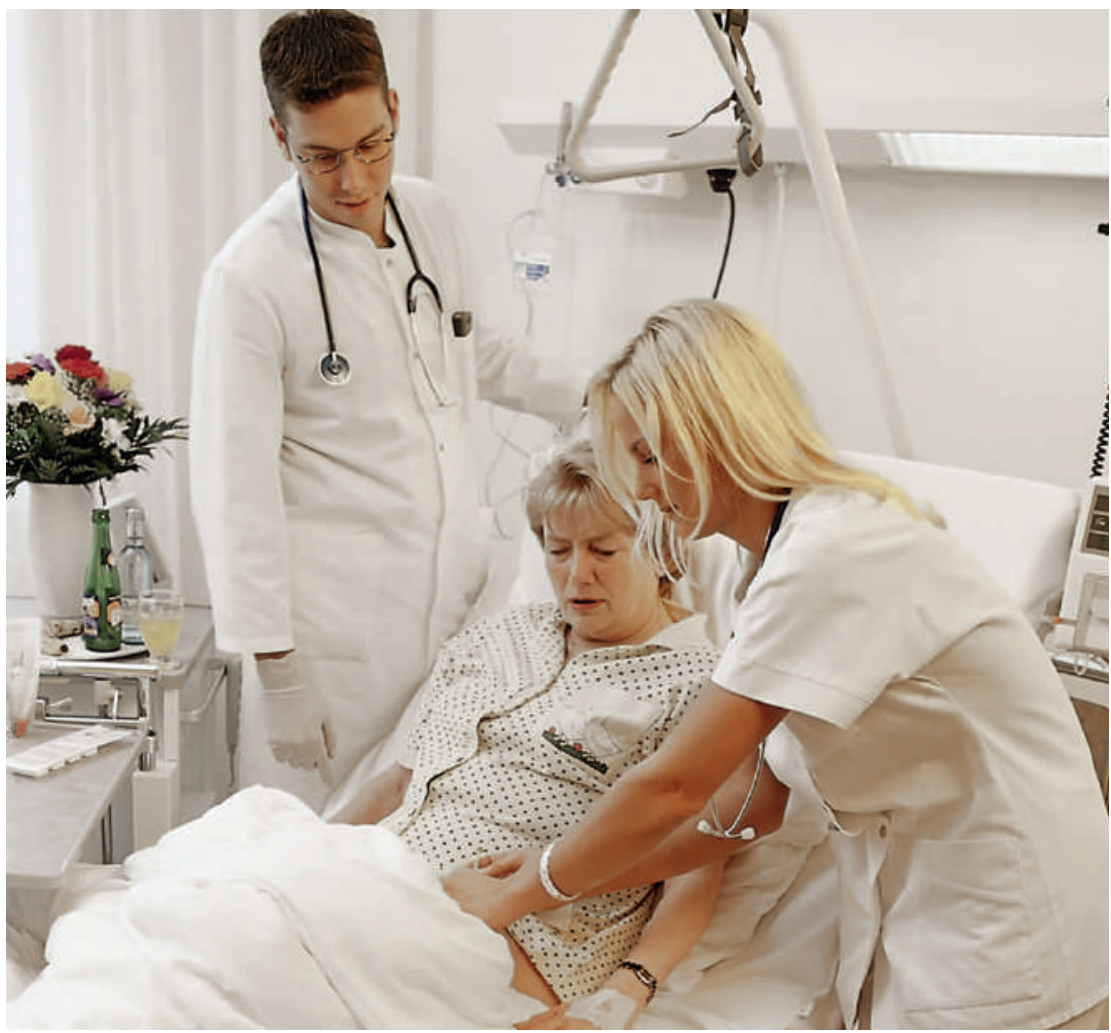

Zufriedenheitsbefragungen bei einem Teil der Kliniken schriftlich und bei einem anderen Teil telefonisch durchgeführt. Dieses führte bei der telefonischen Befragung zu Ergebnissen, die systematisch um 0,25 bis 0,52 Punkte tiefer waren als bei der schriftlichen Befragung, ohne dass dieses ANQ Anlass für eine systematische Korrektur oder angepasste Darstellung gegeben hätte [5].

Die Ergebnisse sind also auch von systematischen Einflüssen abhängig, die nicht primär mit der Behandlung zusammenhängen und nicht immer transparent erkennbar sind.

\section{Risiken und Entwicklungsmöglichkeiten}

Der aus der national transparent erfolgenden Ergebnisveröffentlichung resultierende Druck birgt grundsätzlich die Gefahr, dass Leistungserbringer ihre zukünftigen Aktivitäten in Bezug auf die Patientenzufriedenheit ganz überwiegend auf die Inhalte der fünf zum Einsatz kommenden Fragen beschränken. Dabei muss beachtet werden, dass die Fragen nicht alle relevanten Details erfassen, wobei solche Details jedoch für die Weiterentwicklung der Behandlungsprozesse in Spitälern und Kliniken eine wichtige Bedeutung besitzen. Diese Auffassung vertritt auch die ANQ [7]. Besonders wichtig ist es, die Ergebnisse nicht isoliert zu betrachten, sondern im Kontext von anderen Messungen, beispielsweise der Zuweiserzufriedenheit oder Rückmeldungen aus dem Fehlerund Beschwerdemanagement [14]. Ergebnisse von Spitälern und Kliniken mit weniger als 50 (Akutsomatik) und 30 (Rehabilitation) Befragungsergebnissen wurden nicht in die Auswertung aufgenommen. Dadurch entfielen im Rehabilitationsbereich mehr als ein Viertel der Kliniken [5]. Ein relevanter Anteil der Kliniken erhält also kein Feedback über seine Performance respektive entfällt für den Vergleich mit anderen Spitälern. Gleichzeitig bleibt aus statistischer Sicht anzumerken, dass die Anzahl von Probanden so gering ist, dass der Zufall - oder eine Auswahl der befragten Patienten - einen nicht unerheblichen Einfluss auf das Ergebnis haben könnte.

Obwohl die Forschung um die Patientenzufriedenheit inzwischen sehr umfangreich ist, sind viele der existenten Fragebögen zur Patientenzufriedenheit nicht valide. Eine Übersichtsarbeit von 2006 kommt zum Schluss, dass keines der zur Verfügung stehenden Instrumente zur Erfassung der Patientenzufriedenheit als zufriedenstellend betrachtet werden kann [15]. In diesem Sinne empfiehlt es sich, mit validierten Fragen zu arbeiten. Hierzu gibt ANQ [4] an, dass an der Universität Basel eine Validierung stattgefunden habe. Ein Literaturhinweis findet sich jedoch nicht. Eine Möglichkeit, die Fragen weiterzuentwickeln, wäre die Verwendung von spezifischen

\section{Viele der existenten Fragebögen}

\section{zur Patientenzufriedenheit sind}

\section{nicht valide.}

Modellen und Komponenten aus dem Bereich des Dienstleistungsmarketings, wie dieses andernorts schon vorgeschlagen wurde [16].

Schliesslich ist es wichtig, darauf hinzuweisen, dass Patienten die fachliche Qualität der Leistung nur schwer beurteilen können und somit die Patientenzufriedenheit kein Indikator für die medizinische Qualität ist [8].

\section{Articles interactifs}

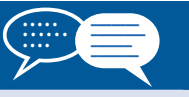

Vous souhaitez commenter cet article? II vous suffit pour cela d'utiliser la fonction «Ajouter un commentaire» dans la version en ligne. Vous pouvez également consulter les remarques de vos confrères sous: www.bullmed.ch/ numero-actuel/articles-interactifs/ 


\section{Literatur}

1 Heidegger T, Saal D and Nuebling M. Patient satisfaction with anaesthesia care: What is patient satisfaction, how should it be measured, and what is the evidence for assuring high patient satisfaction? Best Pract Res Clin Anaesthesiol. 2006;20(2):331-46.

2 Panchaud C, Guillain H, Cranovsky R and Eicher E. Qualitätsterminologie, Schweiz Ärztezeitung. 1999;79(32/33):1960-7.

3 Aharony L and Strasser S. Patient satisfaction: What we know about and what we still want to explore. Medical Care Research and Review. 1993;50:49.

4 Nationaler Verein für Qualitätsentwicklung in Spitälern und Kliniken ANQ, Konzept nationale Patientinnen- und Patientenbefragung, 2011. Heruntergeladen von www.anq.ch/fileadmin/ redaktion/deutsch/Konzept_Nationale Patientenbefragung_def_dt.pdf am 1.11.2014.

5 Wiedenhöfer D, Mathinger N, Schwark B, Müller U, Vouets V and Heller R. Nationale Patientenzufriedenheitsbefragung Rehabilitation, Nationaler Vergleichsbericht Messung 2013 Heruntergeladen von www.anq.ch/rehabilitation/ am 14.11. 2014.

6 Hall JA and Dornan MC. Meta-analysis of satisfaction with medical care: Description of research domain and analysis of overall satisfaction levels, Social Science \& Medicine. 1988;27(6):637-4

7 Wiedenhöfer D, Matzinger N and Müller U. Nationale Patientenbefragung, Nationaler Vergleichsbericht Messung 2012. Heruntergeladen von www. hplusqualite.ch/fileadmin/documents/20140131_ Nationaler_Vergleichsbericht_Patientenbefragung_ 2012_V1_3_final_de.pdf am 1.11.2014.
8 Isaac T, Zaslavsky AM, Cleary PD and Landon BE. The relationship between patients' perception of care and measures of hospital quality and safety. Health Services Research. 2010;45(4):1024-40.

9 Qintana JM, Gonzalez N, Bilbao A, Aizpuru F, Escobar A, Esteban C et al. Predictors of patient satisfation with hospital health care. BMC Health Services Research. 2006;6:102.

10 Kane RL, Maciejewski M and Finch M. The relationship of patient satisfaction with care and clinical outcomes, Medical Care. 1997;35(7):714-30.

11 Hekkert KD, Cihangir S, Kleefstra SM, van den Berg B and Kool RB. Patient satisfaction revisited: A multilevel approach. Soc Sci Med. 2009;69(1):68-75.

12 Sitzia J and Wood N. Response rate in patient satisfaction research: An analysis of 210 published studies, International Journal for Quality in Health Care. 1998;10(4):311-7.

13 Wüthrich-Schneider E, Patientenzufriedenheit: Wie messen? Teil 2: Theoretische Aspekte. Schweiz Ärztezeitung. 2000;81(21):1116-9.

14 Schneider P and Gehrlach S. Qualitätsmanagement: Ein Überblick. SuchtMagazin. 2009;2:38-45.

15 Hawthorne G, Sansoni J, Hayes LM, Marosszeky N and Sansoni E. Measuring patient satisfaction with incontinence treatment. 2006. Centre for Health Service Development, University of Wollongong and the Department of Psychiatry, University of Melbourne.

16. Gill L, White L. A critical review of patient satisfaction. Leadership in Health Services. 2009;22(1):8-19. 